\title{
Application of Differential Evolution for Congestion Management in Power System
}

\author{
Sujatha Balaraman (Corresponding author) \\ Senior Lecturer \\ Electrical Engineering Department, Government College of Engineering \\ Tirunelveli-627007. TamilNadu, India \\ Tel: 91-462-293-0555 E-mail: sujaengg@yahoo.co.in \\ Dr. N.kamaraj \\ Associate Professor \& Head of the Department, Electrical Engineering Department \\ Thiagarajar College of Engineering, Madurai-625015. TamilNadu, India \\ E-mail: nkeee@tce.edu
}

\begin{abstract}
In the emerging restructured power system, the congestion management $(\mathrm{CM})$ has become extremely important in order to ensure the security and reliability of the system. This paper proposes an algorithm for congestion management in a pool based electricity market based on differential evolution (DE). The aim of the proposed work is to minimize deviations from preferred transaction schedules and hence the congestion cost. Numerical results on test system namely IEEE 30 Bus System is presented for illustration purpose and the results are compared with Particle swarm optimization (PSO) in terms of solution quality. The comprehensive experimental results prove that the $\mathrm{DE}$ is one among the challenging optimization methods which is indeed capable of obtaining higher quality solutions for the proposed problem.
\end{abstract}

Keywords: Congestion management, Electricity market, Optimal power flow (OPF), System security, Particle swarm optimization, Differential evolution

\section{Introduction}

The restructuring of the electric power industry has involved paradigm shifts in the real time control activities of the power grids. Managing dispatch is one of the important control activities in a power system. The role of an independent system operator in a competitive market environment is to facilitate the complete dispatch of the power that gets contracted among the market players. With the trend of an increasing number of bilateral contracts being signed for electricity market trades, the possibility of insufficient resources leading to network congestion may be unavoidable. In this scenario, congestion management becomes an important issue. One of the most practiced and an obvious technique of congestion management is rescheduling the power outputs of generators in the system. Other possible means for relieving congestion are operation of phase-shifters or FACTS devices.

\section{Literature Survey}

There are numerous methods proposed in the literature for congestion management. Congestion management for a pool market considering voltage stability is addressed in (Conejo et al.,2006). In (Milano et al., 2003), a multi objective market clearing procedure is presented. Congestion management in open access ensuring voltage stability is proposed by the authors of (Yesturatnam \& Thukaram, 2007). Congestion management based on optimum generation rescheduling and load shedding schemes are reported in (Talukdar et al., 2005; Dutta \& Singh, 2008). Multi objective PSO for congestion management is presented in (Hazra \& Sinha, 2007). The authors of (Kumar et al., 2004) proposed Zonal based congestion management approach .Cluster Based Congestion Management Using PSO is reported in (Meena \& Selvi, 2005). Power transaction using ac power transfer distribution method is discussed in (Kumar \& Srivastava, 2002).Conventional optimization techniques such as gradient-based algorithm, linear programming and interior point methods often have problems of convergence and difficulties in locating the global optima. Since the proposed problem is a complex optimization, use of heuristic algorithm is a vital one. Computational intelligence-based techniques, such as genetic algorithm (GA) and PSO has been successfully used to solve optimization problems in power systems. 
GA represents a particular class of evolutionary algorithms that uses techniques inspired by evolutionary biology such as inheritance, crossover, mutation and natural selection. GA can rapidly locate good solutions, even for difficult search spaces. However the premature convergence of GA degrades its performance and reduces its search capability that leads to a higher probability towards obtaining a local optimum. PSO is an exciting new methodology in evolutionary computation developed by Eberhart and Kennedy in 1995. PSO is not largely affected by the size and nonlinearity of the problem, and can converge to the optimal solution in many problems where most analytical methods fail to converge. References (Mo et al.,2007;Saravanan et al.,2007;Naka et al.,2003) reveal the application of PSO as an optimization tool to various power systems problems.

Differential Evolution is a relatively new member in the family of evolutionary algorithm. It was first proposed by Storn and Price over 1994-1996. Unlike the conventional evolutionary algorithms which depend on predefined probability distribution function for mutation process, DE uses the differences of randomly sampled pairs of objective vectors for its mutation process (Storn \& Price, 1995). DE has drawn an increasing attention for a wide variety of engineering applications including power engineering applications such as transient stability, economic dispatch etc.(Wong \& Dong,2005;Rahnamayan et al.,2008; Cai et al.,2008; Coelho \& Mariani, 2006; Cheng \& Hwang,2001).

In this study an attempt is made to explore the use of differential evolution to determine the optimum generation values for relieving line overloads. However, if congestion cannot be overcome completely by generation rescheduling then load shedding is resorted to. Computer simulations are done on IEEE30 Bus System to validate the potential of proposed algorithm.

\section{Proposed Congestion Management}

When the system is insecure and there are violations in the system, the objective of the pool central dispatcher is to eliminate the system overload and come up with the corrective rescheduling to eliminate the violations as fast as possible. Minimum operating cost, minimum shift from the optimum operation may be used as the objective function.

Notations and Terminology:

$\mathrm{Ng} \quad$ number of generators

Nd number of loads

$N B \quad$ number of buses

$\mathrm{Nl} \quad$ number of lines

$P_{G k}{ }_{C} \quad$ active power produced by generator $k$ as determined by the market clearing procedure

$P_{D j}{ }^{C} \quad$ active power consumed by demand $j$ as determined by the market clearing procedure

$P_{G k} \quad$ final real power generation of generator $k$

$P_{D j} \quad$ final real power consumption of demand $j$

$P_{G k}{ }^{m i n} \quad$ minimum limit for $k^{\text {th }}$ generator real power output

$P_{G k}^{\max } \quad$ maximum limit for $k^{\text {th }}$ generator real power output

$Q_{G k}{ }^{\text {min }} \quad$ minimum limit for $k^{\text {th }}$ generator reactive power output

$Q_{G k}{ }^{\max } \quad$ maximum limit for $k^{\text {th }}$ generator reactive power output

$P_{i j} \quad$ real power flow in line $i-j$

$P_{i j}^{\max } \quad$ maximum flow limit of line $i-j$

$V_{j} \quad \& V_{k}$ Voltage magnitude of bus $j$ and $k$

$\delta_{j \&} \delta_{k} \quad$ Voltage angle of bus $j$ and $k$

$\theta_{k j} \quad$ admittance angle of line $k-j$

$\Delta P_{G k}{ }^{u} \quad$ active power increment of generator $k$ due to congestion management

$\Delta P_{G k}{ }^{d} \quad$ active power decrement of generator $k$ due to congestion management

$C_{k}{ }^{u} \quad$ price bids submitted by generator $k$ to increase its pool power for congestion management

$C_{k}{ }^{d} \quad$ price bids submitted by generator $k$ to decrease its pool power for congestion management

Lo number of overloaded lines

$V B \quad$ number of voltage violated load buses

$p f \quad$ penalty factor

NP non participating generators

BCM before congestion management

ACM after congestion management 
The detailed problem formulation is as follows:

Minimize

$$
T C=\sum_{k \in N g}\left(\begin{array}{c}
u \\
C_{k}^{u} \Delta P G k \\
u
\end{array}+C_{k}^{d} \underset{\Delta P G k}{d}\right) \quad \$ / h r
$$

Where $T C$ is the sum of cost incurred for adjusting real power generation of the participating generators.

Subject to the constraints

$$
\begin{aligned}
& P_{G i}-P_{D i}=V_{i} \sum_{j \in N_{i}} V_{j}\left(G_{i j} \operatorname{Cos} \theta_{i j}+B_{i j} \operatorname{Sin} \theta_{i j}\right) \quad i=1,2, \ldots, N B \\
& Q_{G i}-Q_{D i}=V_{i} \sum_{j \in N_{i}} V_{j}\left(G_{i j} \operatorname{Sin} \theta_{i j}-B_{i j} \operatorname{Cos} \theta_{i j}\right) \quad i=1,2, \ldots, N B \\
& P_{G k}=P_{G k}^{C}+\begin{array}{c}
u \\
\Delta P G k
\end{array}-\underset{\Delta P G k}{d} ; k=1,2, \ldots, N g \\
& P_{D j}=P_{D j}^{C} ; j=1,2, \ldots, N d \\
& P_{G k}^{\min } \leq P_{G k} \leq P_{G k}^{\max }, \forall k \in N g \\
& Q_{G k}^{\min } \leq Q_{G k} \leq Q_{G k}^{\max }, \forall k \in N g \\
& \left(P_{G K}^{C}-P_{G k}^{\min }\right)=\Delta P_{G k}^{\min } \leq \Delta P_{G k} \leq \Delta P_{G k}^{\max }=\left(P_{G k}^{\max }-P_{G k}^{C}\right) \\
& \begin{array}{c}
u \\
\Delta P_{G k}
\end{array} \underset{0 ;}{d} \underset{\Delta P_{G k}}{d} \geq 0 ; k=1,2, \ldots, N g
\end{aligned}
$$

Security constraints:

$$
\begin{aligned}
& P_{i j} \leq P_{i j}^{\max } \\
& V_{i}^{\min } \leq V_{i} \leq V_{i}^{\max }, \quad i \in N L
\end{aligned}
$$

Constraints (2) and (3) represent real and reactive power balances in each node. Constraints (4) and (5) represent final powers as a function of market clearing values. Constraints (6) and (7) provide upper and lower limits for real and reactive power of generators. Constraint (8) provides upper and lower bound for real power adjustment of the participating generators. Constraint (9) ensures that incremental and decremental change in real power of generator as positive. Constraint (10) ensures that the line loading should not exceed its maximum limit. Constraint (11) provides lower and upper bound for load bus voltages. In this study, load bus voltages and real power flow in lines are taken as state variables.

\subsection{Penalized Objective Function}

In this work penalty approach is adopted which penalize the constraints and builds a single objective function, which in turn is minimized by using an unconstrained optimization algorithm. The load bus voltages and real power flow in lines are restricted by adding them as the quadratic penalty terms to the objective function (TC). The penalty objective function (or fitness function) is defined as

$$
\begin{aligned}
& \text { Minimize } F F=T C+p f\left(\sum_{l=1}^{L o}\left(P_{i j}-P_{i j}^{\max }\right)^{2}+\sum_{i=1}^{V B}\left(\Delta V_{i}\right)^{2}\right) \\
& \Delta V_{i}= \begin{cases}\left(V_{i}-V_{i}^{\max }\right) & ; \text { if } V_{i} \geq V_{i}^{\max } \\
\left(V_{i}^{\min }-V_{i}\right) & \text { if } V_{i} \leq V_{i}^{\min }\end{cases}
\end{aligned}
$$


The penalty factor $(p f)$ value should be selected such that in case of congestion, the cost of rescheduling should not be optimum. So the $p f$ is selected as 10000 for the proposed problem.

\section{Differential Evolution Algorithm}

Differential Evolution is presented as a heuristic optimization method which can be used to minimize nonlinear and non-differentiable continuous space functions with real-valued parameters. The optimization process in differential evolution is carried out with three basic operations viz, mutation, crossover and selection. Three essential control parameters involved in DE approaches are scaling factor $(m f)$, crossover constant $(C R)$ and population size $(N p)$.The scaling factor is a value in the range $(0,2)$ that controls the amount of perturbation in the mutation process. The crossover constant is a value in the range $(0,1)$ that controls the diversity of the population. The population size determines the number of individuals in the population and provides the algorithm with enough diversity to search the solution space (Gamperie \& Muller,2002).

\subsection{Notations:}

$\begin{array}{ll}N p & \text { population size } \\ n c & \text { number of control }\end{array}$

$n c \quad$ number of control variables

$k \quad$ iteration count

$k_{\max } \quad$ maximum iteration

$Y_{i} \quad i^{\text {th }}$ individual of a parent population $Y$

$Y_{i, j} \quad j^{\text {th }}$ element in $i^{\text {th }}$ individual of $Y$

$M_{i} \quad i^{\text {th }}$ mutant vector of population

$T_{i} \quad i^{\text {th }}$ trial vector of population

mf mutation factor (constant)

$C R \quad$ crossover rate (constant)

$F F\left(Y_{i}\right)$ fitness function value of $i^{\text {th }}$ parent population

$F F^{*}$ best fitness function value

$X^{*} \quad$ best solution vector in $k^{\text {th }}$ iteration

\subsection{Pseudo code}

Step 1:Set iteration $k=1$

For $i=1$ to $N p$

For $j=1$ to $N c$

Randomly generate initial parent population $Y_{i j}$ and compute fitness values $F F_{i}$. Each individual parameters or vectors of the population is called target vector.

end

end

Set $F F^{*}$ and store corresponding $Y_{i j}$ in $X^{*}$.

Step 2: Mutation

For $i=1$ to $N p$

Compute mutant vectors $M$ using (14).

$$
M_{i}{ }^{(k+1)}=Y_{a}{ }^{(k)}+m f \cdot\left(Y_{b}^{(k)}-Y_{c}^{(k)}\right)
$$

Where $a$ and $b$ are randomly selected individual such that $a \neq b \neq c \neq i$ and $a, b, c, i \quad \epsilon\left\{1, \quad N_{p}\right\}$

end

Step 3: Cross over operation

Combine parent vectors $Y_{i}^{(k)}$ and mutant vectors $M_{i}^{(k+I)}$ and select $N_{p}$ trial vectors from $2 . N_{p}$ vectors by using (15)

For $i=1$ to $N_{p}$

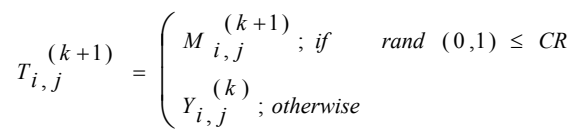

end 
Compute fitness function values $F F_{i}$ for trial vector $T_{i, j}{ }^{(k+1)}$.

Step 4: Selection process

For $i=1$ to $N_{p}$

Compare the fitness function values of each trial vector $T_{i}^{(k+1)}$ and parent vector $Y_{i}^{(k)}$. The vector with best fit is stored as

$$
Y_{i}^{(k+1)}=\left\{\begin{array}{l}
T_{i}^{(k+1)} ; \text { if fit }\left(T_{i}{ }^{(k+1)}\right) \leq f i t\left(Y_{i}^{(t)}\right) \\
Y_{i}^{(k)} ; \text { otherwise }
\end{array}\right.
$$

end.Update $F F^{*}$ and store the corresponding $Y_{i j}$ in $X^{*}$.

Step 5: $\quad$ Stopping Criteria:

If $k>k_{\max }$, then report the best fitness function value $F F^{*}$ and the corresponding solution $X^{*}$ and terminate the program. Otherwise set $k=k+1$ and go to step 2.

\subsection{Application of DE for the proposed problem}

The following describes the incorporation of DE algorithm into the proposed problem .

Step1: Input system data, parameters of DE.

Step 2: Set iteration count $k=1$ and maximum iteration as $k_{\max }$.

Step3: Generate parent vectors randomly within the upper and lower bounds

${ }_{i}^{Y_{i}^{k}=\left(\Delta P_{G i j}^{+}\right.}{ }^{+}$and $\left.\Delta P_{G i j}^{-} ; i=1,2, \ldots N p, j=1,2, \ldots, N g\right)$. For each individual run Newton Raphson (NR) power

flow and compute slack bus power and check for limit violation if any. If it violates the

operational limit then the corresponding particle is regenerated.

Step 4. Store the fittest member of the Differential Evolution in vector $X^{*}$.

Step 5. For each parent vector $Y_{i}^{k}$, generate a candidate child vector $M_{i}^{(k+1)}$ based on the distance

of two other parameter vectors using (14).

Step 6. Generate trial vector $T_{i}^{(k+1)}$ from parent vector and child vector using cross over operation (15).

Step 7. Selection is the procedure of producing better off spring. To decide whether or not the vector

$T_{i}^{(k+1)}$ should be a member of the population comprising the next generation, compare its fitness

value with the fitness value of the corresponding vector $Y_{i}^{k}$ by using (16). The vector with

best fit is stored as $Y_{i}^{(k+1)}$.

Step 8. Evaluate fitness values for $Y_{i}^{(k+1)}$.Update vector $X^{*}$. Check for line congestion. If the congestion is relieved completely or if the maximum iteration is reached, then go to next step. Otherwise set $k=k+1$ and go to step 5 .

Step 9.Print the best value of $X^{*}$ as optimal solution.

\section{Results and Discussion}

For examining the proposed approach, IEEE 30 Bus system is tested. This system consists of 6 generator buses, 24 load buses and 41 transmission lines. System data are taken from (Pai,1979). Price bids submitted by Generation companies (GENCOs) to alter their scheduled productions from initial market clearing values are reported in Table 1. Generator upcost is taken slightly more than the corresponding marginal cost value and the downcost is taken slightly less than the marginal cost (Conejo et al., 2006). The proposed approach is illustrated through corrective rescheduling for a few harmful contingencies and the same has been implemented using Matlab 7.4.

Details of case studies considered in this study are given in Table 1. In Case A, total load of the system is raised by $40 \%$, in Case B the unavailability of line 1-3 along with increase in total load by $25 \%$,in Case C outage of line 1-2 and in Case D, total load of the system is raised by $50 \%$ along with unavailability of line 1-3. In each Case, number of lines get overloaded and their associated power flows are determined by conducting full a.c 
power flow and they are listed in Table 1.

DE parameters such as $m f$ and $C R$ are selected through experiment. For different combinations of parameters, the proposed problem is carried out with 20 particles and maximum of 300 iterations. For each set of parameters, 25 independent trials were carried out and the best value of objective function (TC) obtained are summarized in Table 2.

From Table 2, it is clear that $m f=0.7$ and $C R=0.8$ gives best result, therefore these parameters are used throughout the simulations. Also the optimum number of particles in the population is found to be 20 through experiment. PSO parameters such as cognitive and social parameters $(C 1, C 2)$, inertia weight $\omega_{\min }$ and $\omega_{\max }$ are also selected through experiment. Table 3 shows the optimal parameter settings for DE and PSO. These values have been selected by solving the proposed optimization problem with different combinations of parameters for 25 independent trials.

In case A, line 1-2 get overloaded by $35.33 \%$ due to increase in load by $113.36 \mathrm{MW}$ and $50.48 \mathrm{MVAR}$ (Table 1 ). The objective is to minimize the rescheduling of generation required to limit the flow in line $1-2$ by $130 \mathrm{MW}$. The proposed method effectively relieve overload with minimum number of generators whereas the PSO method yield optimum solution considering all the generators (Table 4). The proposed method yield good quality solution with minimum cost of $2630.7 \$ / \mathrm{hr}$ than PSO in Case A.

Figure 1 shows the convergence of fitness values for DE and PSO. It is clear from figure that DE algorithm has good searching capability. Also the rate of convergence is faster than PSO. DE yields high quality solution in the earlier iteration of less than 140, after which there is no significant variations in fitness value. Though PSO converges in earlier iteration, the solution yielded by PSO is not a global one.

In Case B three lines get congested viz. 1-2, 2-4 and 2-6. The amount of power violation is found to be 116.52 MW. Optimal power adjustment required for congestion management is shown in figure 2 . The proposed method gives economical solution with total cost $(T C)$ of $2598.6 \$ / \mathrm{hr}$ for relieving congestion in the above said lines.

Amount of power rescheduling and the total cost incurred for congestion management (CaseA-CaseD) are summarized in Table 5. The total cost incurred for relieving the congestion is considered as revenues for the GENCOs for their participation in congestion management. The solutions reported in Table 5 not only relieve congestion but also reduce system losses. The real and reactive power losses before congestion management and after congestion management are shown in figure 3. Through Case studies it is proved that DE approach yield high quality solution (i.e. minimum congestion cost) than PSO for all the test Cases.

To investigate the effects of the initial trial solutions on the objective function values, different initial trial solutions are given to PSO and DE approaches. The best solution achieved for relieving congestion (Case D) with 25 different initial trials are shown in figure 4. The solutions yielded by PSO are scattered with in a band of $+/-52 \%$, where as the solutions obtained from DE are scattered within a narrow band of $+/-1 \%$ for entire trials. From the experiment it is revealed that the proposed DE is very efficient and robust algorithm for solving non linear optimization problems, which yield consistent solution in each trial and hence it is suggested for the proposed Congestion management problem.

\section{Conclusion}

This paper focuses on congestion management by corrective rescheduling in the context of power system restructuring using differential evolution. Due to the population of candidate solutions, the proposed algorithm is capable of determining the global optimum solution in the presence of multiple local optima. Feasibility and robustness of the proposed method is tested on IEEE 30 Bus system. The results of case studies have shown that, $\mathrm{DE}$ would be an effective tool in handling transmission congestion in deregulated environment and results in secure operating condition. The proposed model can be extended to any practical network.

\section{References}

Cai H.R., Chung C.Y., \& Wong K.P. (2008). Application of Differential Evolution Algorithm for Transient Stability Constrained Optimal Power Flow. IEEE Transactions on power systems., 23(2),719-728.

Cheng, S.L., \& Hwang, C. (2001). Optimal Approximation of Linear Systems by a Differential Evolution Algorithm. IEEE Transactions on Systems, man, and cybernetics-part a: systems and humans, 31(6), 698-707.

Coelho.L.D.S., \& Mariani.V.C. (2006). Combining of Chaotic Differential Evolution and Quadratic Programming for Economic Dispatch Optimization With Valve-Point Effect. IEEE Transactions on Power Systems., 21(2),989- 996.

Conejo,J.A., Milano,F.,\& Bertrand ,R.G. (2006). Congestion management ensuring voltage stability.IEEE Trans. Power Syst., 21(1),357-364. 
Dutta,S., \& Singh, S.P. (2008). Optimal Rescheduling of Generators for Congestion Management based on Particle Swarm Optimization. IEEE Trans. Power Syst., 23 (4), 1560- 1569.

Gamperie, R. \&, Muller,S. (2002). A parameter study for Differential Evolution. Advances in Intelligent systems, Fuzzy systems, Evolutionary computation, WSEAS Press., 293 - 298.

Hazra,J.,\& Sinha,K.A. (2007). Congestion Management using multi objective Particle Swarm Optimization. IEEE Trans Power Syst., 22(4), 1726-1734.

Kumar,A., \& Srivastava, S.C. (2002). AC Power Transfer Distribution Factors for Allocating Power Transactions in a Deregulated Market. IEEE Power Engineering Review., 42-43.

Kumar, A., Srivastava, S.C., \& Singh, S.N. (2004). A zonal Congestion Management Approach using real and Reactive Power Rescheduling. IEEE Trans. Power Syst., 19(1), 554-562.

Meena,T., \& Selvi,K. (2005). Cluster Based Congestion Management in Deregulated Electricity Market Using PSO.IEEE Indicon Conference, Chennai,India,11-13, 627-630.

Milano, F., Cañizares ,C. A., \& Invernizzi, M. (2003). Multiobjective optimization for pricing system security in electricity markets. IEEE Trans.Power Syst., 18(2),596- 604.

Mo.N., Zou,Z.Y., Chan,K.W., \& Pong, T.Y.G. (2007). Transient stability constrained optimal Power flow using Particle Swarm Optimization.IET Gener.,Transm.,Distrib.,1(3),476-483.

Naka, S., Genji,T., Yura,T., \& Fukuyama, Y. (2003). A hybrid particle swarm optimization for distribution state Estimation. IEEE Trans. Power Syst., 18(1), 60-68.

Pai,M.A.(1979).Computer Techniques in Power System Analysis.Mc Graw-Hill, New Delhi.

Rahnamayan, S., Tizhoosh,H.R., \& Salama,M.M.A. (2008). Opposition- Based Differential Evolution. IEEETransactions on evolutionary computation., 12( 1),64-79.

Saravanan, M., Slochanal,S.M.R., Venkatesh,P., \& Abraham,J.P.S. (2007). Application of particle Swarm Optimization technique for optimal location of FACTS devices considering cost of installation and system load ability. Electrical Power System Research.,77, 276- 283.

Storn,R., \& Price,K.(1995).Differential Evolution- A Simple and efficient adaptive scheme for Global optimization over continuous spaces .Berkeley, CA,tech.Rep.TR-95-012.

Talukdar, B.K., Sinha, A.K., Mukhopadhyay, S., \& Bose, A. (2005). A computationally simple method for cost-efficient generation rescheduling and load shedding for Congestion Management. Electrical Power and Energy Systems., 27,379-388.

Wong,K.P., \& Dong,Z.Y. (2005). Differential Evolution, an Alternative Approach to Evolutionary Algorithm.ISAP, 73-83.

Yesuratnam, G., \& Thukaram,D. (2007). Congestion Management in open access based on relative electrical distances using voltage stability criteria. Elect. Power Syst. Res.,77, 1608-1618. 
Table 1. Simulated Cases

\begin{tabular}{|c|c|c|c|c|c|c|c|}
\hline \multirow{2}{*}{$\begin{array}{l}\text { Gen. } \\
\text { No. }\end{array}$} & \multicolumn{2}{|c|}{$\begin{array}{l}\text { Price Bids submitted by } \\
\text { GENCOs }(\$ / M W h r)\end{array}$} & \multirow{2}{*}{ Case } & \multirow{2}{*}{ Type } & \multirow{2}{*}{ Line } & \multirow{2}{*}{$\begin{array}{l}\text { Line flow } \\
\text { limit } \\
\text { (MW) }\end{array}$} & \multirow{2}{*}{$\begin{array}{l}\text { Total powe } \\
\text { violation } \\
\text { (MW) }\end{array}$} \\
\hline & $C_{k}^{u}$ & $C_{k}^{d}$ & & & & & \\
\hline 1 & 22 & 18 & A & $\begin{array}{l}\text { Total load raised by } \\
40 \%\end{array}$ & $1-2$ & 130 & 45.935 \\
\hline \multirow[t]{2}{*}{2} & \multirow{2}{*}{21} & \multirow[t]{2}{*}{19} & \multirow{3}{*}{ B } & \multirow{3}{*}{$\begin{array}{l}\text { Outage of the line } \\
1-3 \text { and total load } \\
\text { raised by } 25 \%\end{array}$} & $1-2$ & 130 & \multirow{3}{*}{116.52} \\
\hline & & & & & $2-4$ & 65 & \\
\hline 3 & 42 & 38 & & & $2-6$ & 65 & \\
\hline \multirow[t]{2}{*}{4} & \multirow{2}{*}{43} & \multirow[t]{2}{*}{37} & \multirow{2}{*}{$\mathrm{C}$} & \multirow{2}{*}{$\begin{array}{l}\text { Outage of the line } \\
1-2\end{array}$} & $1-3$ & 130 & \multirow{2}{*}{23.393} \\
\hline & & & & & $3-4$ & 130 & \\
\hline 5 & 43 & 35 & \multirow{3}{*}{$\mathrm{D}$} & \multirow{3}{*}{$\begin{array}{l}\text { Outage of the line } \\
1-3 \text { and total load } \\
\text { raised by } 50 \%\end{array}$} & $1-2$ & 130 & \multirow{3}{*}{251.072} \\
\hline \multirow[t]{2}{*}{6} & \multirow{2}{*}{41} & \multirow[t]{2}{*}{39} & & & $2-4$ & 65 & \\
\hline & & & & & $2-6$ & 65 & \\
\hline
\end{tabular}

Table 2. Effects of Variation of DE Parameters on objective function- Case A

\begin{tabular}{|c|c|c|c|}
\hline S.No & $m f$ & $C R$ & Best Cost $(\$ / h r)$ \\
\hline 1 & 0.3 & 0.6 & 2639.2 \\
\hline 2 & 0.3 & 0.8 & 2634.2 \\
\hline 3 & $\mathbf{0 . 7}$ & $\mathbf{0 . 8}$ & $\mathbf{2 6 3 0 . 7}$ \\
\hline 4 & 1.0 & 0.9 & 2632.1 \\
\hline 5 & 0.3 & 0.4 & 2638.0 \\
\hline 6 & 0.7 & 0.7 & 2635.7 \\
\hline 7 & 0.5 & 0.5 & 2639.9 \\
\hline
\end{tabular}

Table 3. Best parameter settings for DE and PSO

\begin{tabular}{|c|c|}
\hline DE & PSO \\
\hline Population Size: 20 & Population Size: 20 \\
\hline Mutation factor $=0.7$ & $\omega_{\max }=0.9 \& \omega_{\min }=0.4$ \\
\hline Cross over rate $=0.8$ & $C 1=\mathrm{C} 2=2.0$ \\
\hline Maximum iterations $=300$ & Maximum iterations $=300$ \\
\hline
\end{tabular}

Table 4. Contribution of generators for congestion management- Case-A

\begin{tabular}{|c|c|c|c|c|c|c|}
\hline \multirow{2}{*}{ Method } & \multicolumn{3}{|c|}{ Up/down adjustment of the participating } & \multicolumn{3}{c|}{ generators (MW) } \\
\cline { 2 - 7 } & $\Delta \mathrm{P}_{\mathrm{G} 1}$ & $\Delta \mathrm{P}_{\mathrm{G} 2}$ & $\Delta \mathrm{P}_{\mathrm{G} 3}$ & $\Delta \mathrm{P}_{\mathrm{G} 4}$ & $\Delta \mathrm{P}_{\mathrm{G} 5}$ & $\Delta \mathrm{P}_{\mathrm{G} 6}$ \\
\hline $\mathrm{PSO}$ & +67.0 & +37.69 & +2.863 & +2.686 & +0.365 & +11.892 \\
\hline $\mathrm{DE}$ & +51.02 & +71.81 & $\mathrm{NP}$ & $\mathrm{NP}$ & $\mathrm{NP}$ & $\mathrm{NP}$ \\
\hline
\end{tabular}


Table 5. Performance Comparisons of PSO and DE

\begin{tabular}{|c|c|c|c|c|c|c|c|c|}
\hline \multirow[t]{2}{*}{ Case } & \multicolumn{3}{|c|}{$\begin{array}{l}\% \text { Loading of } \\
\text { line after } \\
\text { congestion } \\
\text { relieving }\end{array}$} & \multicolumn{2}{|c|}{$\begin{array}{l}\text { Amount of generation } \\
\text { rescheduling for } \\
\text { congestion } \\
\text { management (MW) }\end{array}$} & \multicolumn{2}{|c|}{$\begin{array}{c}\text { Total } \\
\text { congestion } \\
\text { Cost }(\$ / \mathrm{hr})\end{array}$} & \multirow[t]{2}{*}{$\begin{array}{c}\text { Cost } \\
\text { Saving } \\
(\%) \text { by } \\
\text { proposed } \\
\text { method }\end{array}$} \\
\hline & line & $\mathrm{PSO}$ & DE & PSO & DE & PSO & DE & \\
\hline A & $1-2$ & 100 & 86.91 & 122.496 & 122.83 & 3004.7 & 2630.7 & 14.22 \\
\hline \multirow[t]{3}{*}{ B } & $1-2$ & 99.9 & 100 & \multirow[t]{3}{*}{93.154} & \multirow[t]{3}{*}{93.776} & \multirow[t]{3}{*}{2705.4} & \multirow[t]{3}{*}{2598.6} & \multirow[t]{3}{*}{4.11} \\
\hline & $2-4$ & 96.3 & 95.5 & & & & & \\
\hline & $2-6$ & 100 & 99.98 & & & & & \\
\hline \multirow[t]{2}{*}{$\mathrm{C}$} & $1-3$ & 99.9 & 100 & \multirow{2}{*}{22.936} & \multirow{2}{*}{23.014} & \multirow{2}{*}{538.95} & \multirow{2}{*}{457.694} & \multirow{2}{*}{17.75} \\
\hline & $3-4$ & 92.9 & 92.92 & & & & & \\
\hline \multirow{3}{*}{ D } & $1-2$ & 99.7 & 100 & \multirow{3}{*}{168.03} & \multirow{3}{*}{167.746} & \multirow{3}{*}{5335.5} & \multirow{3}{*}{5290.8} & \multirow{3}{*}{0.84} \\
\hline & $2-4$ & 94.0 & 98.74 & & & & & \\
\hline & $2-6$ & 99.4 & 100 & & & & & \\
\hline
\end{tabular}

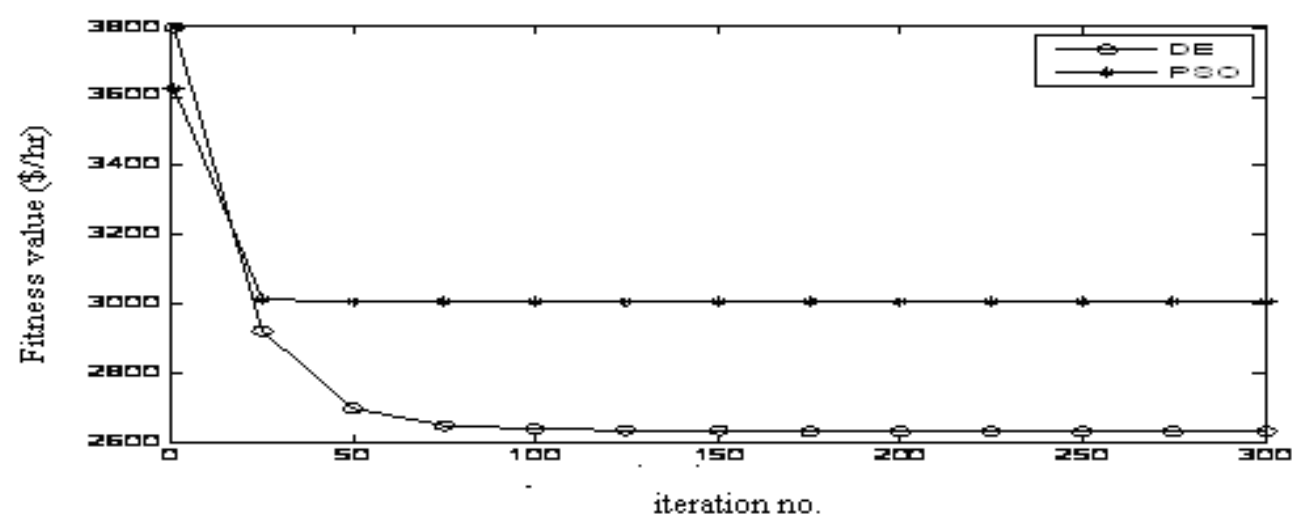

Figure 1. Fitness Convergence characteristics of DE and PSO of Case A

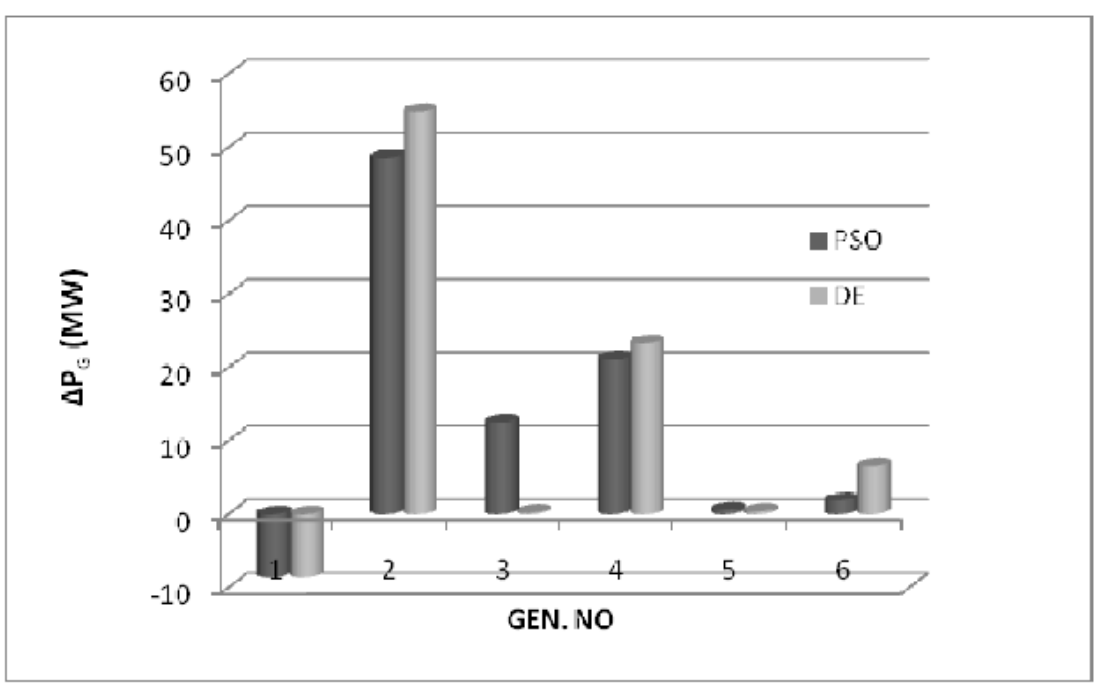

Figure 2. Contribution of generators for congestion management- Case B 


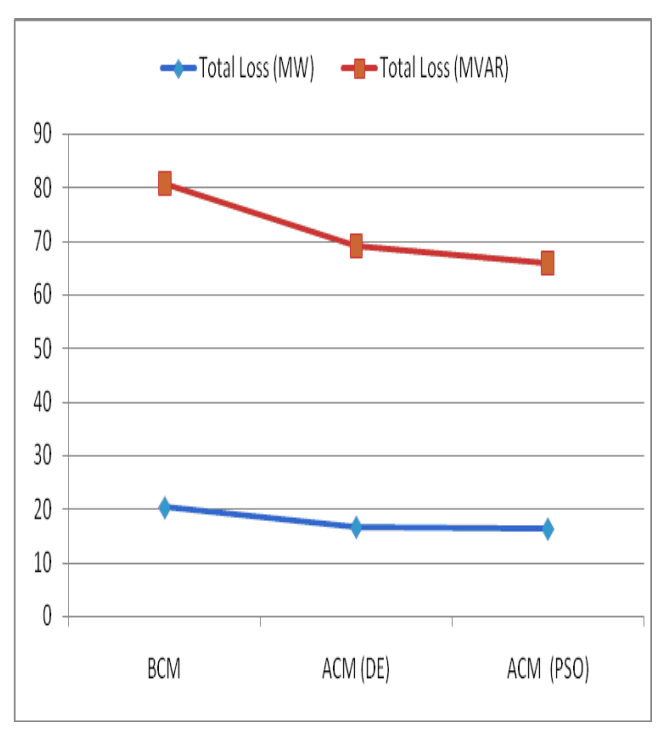

Figure3a. Case A

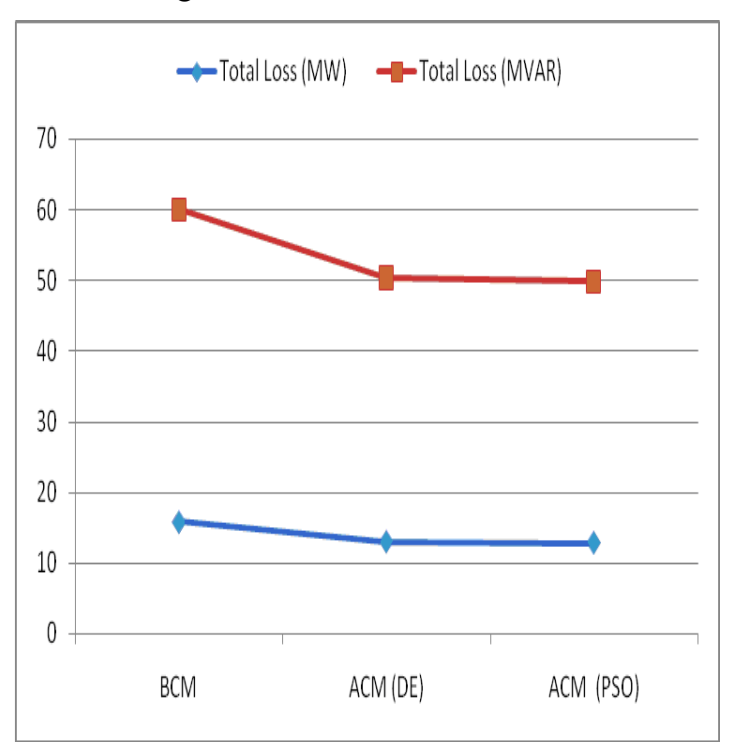

Figure 3c. Case C

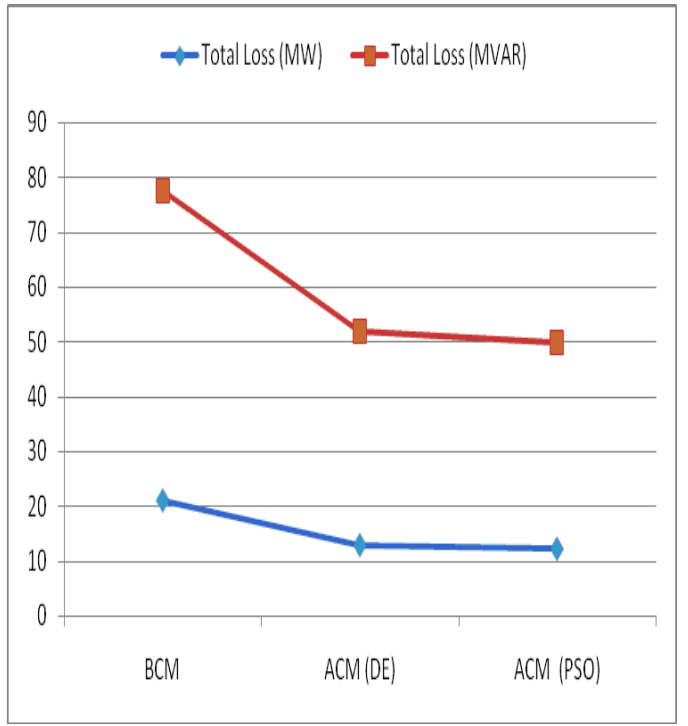

Figure 3b.Case B

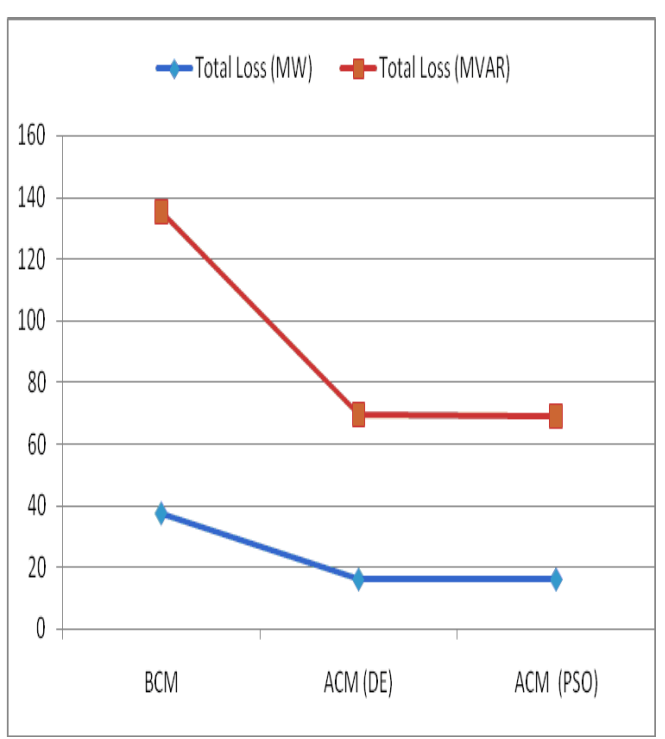

Figure 3d. Case D

Figure 3. Comparisons of Real and Reactive Power Loss for various Cases

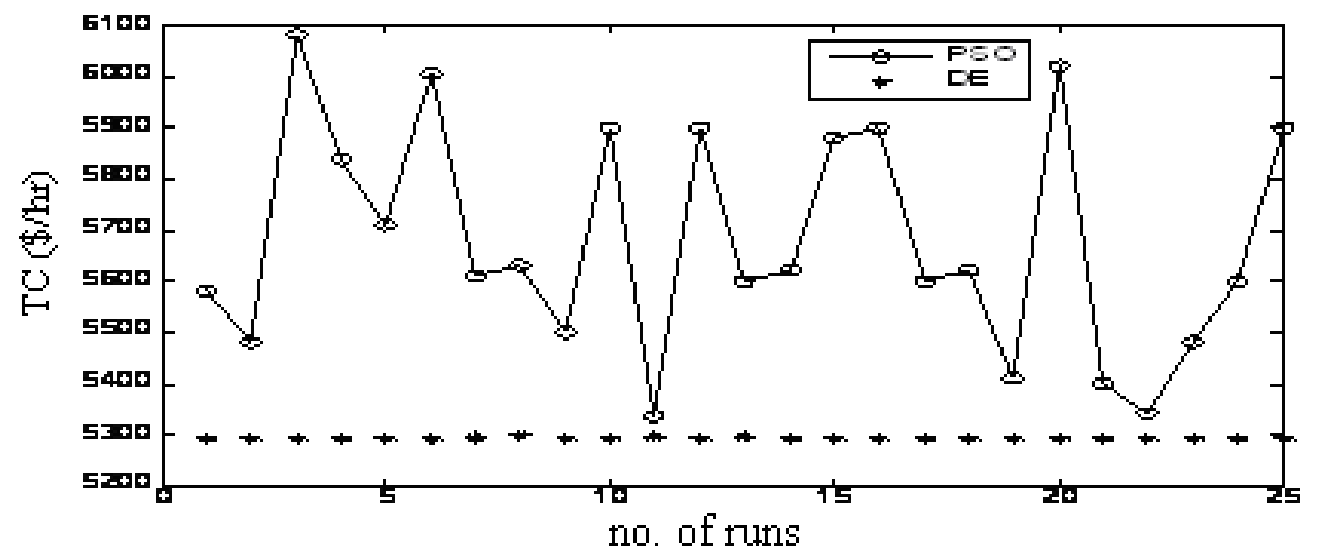

Figure 4. Cost Distribution of final solutions with 25 different initial trials-Case D 